\title{
Revenue management systems as symbiotic analytics systems: insights from a field study
}

\author{
Claudia Schütze ${ }^{1}$ - Catherine Cleophas $^{2}$ (D) \\ Monideepa Tarafdar ${ }^{3}$
}

Received: 15 October 2019/Accepted: 6 August 2020/Published online: 12 August 2020

(C) The Author(s) 2020

\begin{abstract}
Revenue management is a complex operational planning process involving predictive and prescriptive analytics. As real-world implementations strongly rely on the joint outcomes from both algorithms and analysts, we consider the revenue management system as an example of symbiotic analytics systems. This paper presents insights from a field study observing a natural experiment in revenue management. As a firm updates its automated revenue management systems, it also updates the related processes and the corresponding organizational structure. We use this opportunity to examine the multilevel use of symbiotic analytics systems based in a field study and explore the implications for the design of future systems. Specifically, we identify two different perspectives on the revenue management process. In the functional view, jobs are organized sequentially with a high degree of system-oriented specialization. The process view organizes jobs in a parallel structure, differentiating two perspectives on demand. Depending on what view the firm implements, different structural fault lines turn the communication and training of analysts into keystones of the planning process. Furthermore, as we point out, even implementing more sophisticated algorithms and redesigning planning processes and organization do not seem to reduce the relevance of human analysts.
\end{abstract}

Catherine Cleophas

cleophas@bwl.uni-kiel.de

Claudia Schütze

claudia.schuetze@rwth-aachen.de

Monideepa Tarafdar

m.tarafdar@lancaster.ac.uk

1 School of Business and Economics, RWTH Aachen University, Aachen, Germany

2 Service Analytics, CAU Kiel University, Kiel, Germany

3 Management School, Lancaster University, Lancaster, UK 
Keywords Revenue management $\cdot$ Symbiotic analytics $\cdot$ Field study $\cdot$ Natural experiment

\section{Motivation}

The importance of analytics and the role of algorithms in general and artificial intelligence for business operation in particular is growing (Choi et al. 2018). This development increases the urgency understanding the interplay of human analysts and automated systems in the context of the use of information systems (IS). As an exemplary area of analytics, this paper analyzes the revenue management (RM) process in a field study featuring an airline updating its systems and processes. In that, it focuses on the combination of automated algorithms and analyst interventions.

"RM aims at selling the right product to the right customer at the right time and the right price" (Smith et al. 1992). It lets firms maximize expected revenue by selling a fixed capacity of perishable products under uncertain and heterogeneous demand. The concept is a relevant example of analytics, as it incorporates descriptive, predictive, and prescriptive methods for estimating, forecasting, and managing demand.

RM is crucial for many service industries, such as hotels, car rentals, and airlines. Airlines, in particular, are frequently seen as the cradle of the methodology (Horner 2000). In many transport industries, strategic and tactical planning fix the capacity through scheduling and fleet assignment. As marginal costs are regarded as negligible, transport companies can maximize revenue in an operational planning step, optimally selling a given capacity of seats over a fixed booking horizon. The field study presented here features a capacity-based variant of RM. In this variant, an optimization component considers the forecasted demand and the given capacity to compute the best set of fare classes to offer at any time in the booking horizon.

Considering the advanced state of RM from the perspective of operations research, one could conclude that the topic is firmly in the hands of automated IS. Firms implement such automated RM systems at a significant financial and organizational effort. Nevertheless, they ensure that human analysts continuously monitor and control the automated system's output. In fact, analysts, rather than algorithms, are regarded as being responsible for the firm's revenue success (Zeni 2003). This turns RM into a complex interaction of analysts and systems, consisting of both mathematical algorithms and human supervision and intuition.

The role of analysts causes some authors to define RM systems as decision support systems (DSS). However, DSS aim to support and improves decisionmaking on a managerial level and are primary used for unstructured and underspecified problems, often of a strategic nature (compare Arnott and Pervan 2005; Sprague 1980). In contrast, we argue that RM does not consist of "nonroutine, episodic situations that require judgment" that DSS aim to support (Kasper 1996). Instead, the vast majority of RM decisions are routine, repetitive, and highly frequent, taking place on an operational level, so that no fully manual implementation is conceivable. Given this and the balance of analyst input and algorithmic 
computation, we consider RM an example of what we term symbiotic analytics systems (SAS). Further examples from the domain of operational planning that combines algorithms and analysts to implement predictive and prescriptive analytics are inventory management (Wild 2017), work-shift scheduling (Lodree et al. 2009), and assortment optimization (Hart and Rafiq 2006). SAS would not function without automated algorithms, as human decision makers alone cannot prepare, make, and implement the high number of operational decisions. For example, RM as implemented at the airline featured in the field study controls ticket offers for more than 6000 flight departures per day. SAS cannot, in practice, function without the work of human experts either. In RM, analysts monitor bookings, adjust the forecast, set the parameters for the optimization of offers and overbookings, and override inventory controls. While extensive existing research focuses on the underlying mathematical models, we argue effects from integrating analytics algorithms into the firm's processes and particularly the role of analysts, who adjust and overwrite parameters and results, require further examination.

As a contribution to opening up this research area, we analyze the RM process in a field study by observing a firm updating its RM methodology through a switch in software, which also triggers changes in the organizational structure. We apply a qualitative approach to draw a meaningful picture of the interconnection of RM systems and analyst organization using qualitative data from interviews and archival company documentation. Our aim is to show a lively picture of the tasks, decisionmaking and challenges an analyst has to face rather than a quantitative measuring of e.g. amount of system usage.

As Leonardi and Barley (2008) state, technology and the organization are both important, but the way they are connected is not yet fully understood. The field study offers the unique opportunity to observe a firm moving toward a new automated RM system and adjusting its processes and analyst responsibilities to fit the new methodology. As stated by Orlikowski (2001), information technology research and organization research can learn from each other as the technology is applied in real-world organizational structures. Therefore, this paper examines how the RM technology is implemented in the organizational structure of analysts' work. Our research is also motivated by Liozu and Hinterhuber (2014), who emphasize the importance of pricing capabilities for firms and highlights that further research in the organizational aspect of these capabilities is necessary, especially on the role of individual actors. Therefore, we aim to shed light on the interplay of the automated system, individual analysts, and the organization.

In the next section, we review existing research considering the RM domain from a perspective that is not solely focused on introducing new models or algorithms. Subsequently, we introduce the theoretical background on multilevel analysis and task analysis applied to the field study. We apply this theory to the general model of RM outlined in this introduction, we document and discuss insights from the field study. Finally, we conclude the paper and give an outlook on further research in the final section. 


\section{Existing research}

The vast majority of RM contributions focuses on the problem's mathematical aspects from the perspective of operations research. The standard textbook of Talluri and Ryzin (2004) supplies a wide overview of such literature. Both Talluri and Ryzin (2004) and recent considerations, e.g. of allowing RM to deal with uncertainty (Gönsch 2017; Cleophas et al. 2017), emphasize the relation of RM and analytics (Evans and Lindner 2012). RM systems embody multilevel approaches of analytics by describing the current state of offers and bookings, predicting future demand, and prescribing future offers. As uncertain and dynamic market environments make it difficult to perfectly predict demand fluctuations and motivate business objectives beyond pure short-term revenue, RM systems also strongly rely on the work of human analysts. As acknowledged in contributions such as Mukhopadhyay et al. (2007) and Currie and Rowley (2010), firms frequently task analysts with complementing the forecast based on information on local and global events and economic factors. This idea is related to the field of judgmental forecast adjustments as surveyed in Arvan et al. (2019). Besides, as pointed out by Che-Ha et al. (2014) and Cleophas et al. (2017), analysts can adjust the results of the automated optimization. Here, the idea is that such adjustments support the system's market orientation by aligning its outcomes with the firm's goals.

In relying on this interplay, RM systems as symbiotic analytics systems embody an idea that Ghallab et al. (2014), focusing on artificial intelligence, differentiate as "planning" a solution that could be immediately implemented rather than "enacting" it. The authors highlight that acting requires deliberation and continuous reasoning and point out opportunities for future research on artificial intelligence to incorporate this reasoning within the automated system.

An emerging strain of research focuses on behavioral perspective of human decision making when facing the RM problem without emphasizing the role of an automated system [compare Bearden et al. (2008), Bendoly (2011, 2013), Kocabiyikoglu et al. (2015, 2018), Schütze and Cleophas (2019)]. These studies focus on individual behavior in a revenue management setting. Main findings in this stream of research are that participants use advanced decision policies (Bearden et al. 2008) but the number of simultaneous tasks increases stress and faulty decisions (Bendoly 2011). Furthermore, participants reserve more units of capacity for high-value customers when they set protection levels instead of bookings levels (Kocabiyikoglu et al. 2018), and they anchor their decisions on non-stationary information like the willingness to pay of customers (Schütze and Cleophas 2019). At most, these studies consider a forecast as given to support human decision making. Such a given forecast follows the idea of differentiated RM process steps, but no further consideration of job division or hierarchical decision making is included in the experimental designs.

Dutta et al. (2003) conduct a field study focusing on pricing and discounting endcustomer contracts. They conclude that pricing is an important capability and that having a pricing system that allows for flexible and accurate price setting is a crucial success factor. From a macro-perspective on strategic and organizational decision 
making in RM, Fürstenau et al. (2019) analyze a field study where a firm struggles to implement novel pricing capabilities. The authors find that existing market standards make it difficult to radically change the approach to pricing from capacity-based RM to dynamic pricing.

IS research on system usage and fit focuses, for example, on organizationalsystem fit and possible misfits [compare Strong and Volkoff (2010)], the fit of organizational culture and IS [compare Wang and Yeoh (2009), Wanyama and Zheng (2011)], or on the effectiveness of information systems usage [compare Burton-Jones and Grange (2013)]. While these research topics also open up an interesting research opportunity when applied to RM, we focus on a different perspective. Instead of measuring the fit of RM systems to existing organizational variables, we focus on different possible interconnections of IS, users, and organizational structure that can be implemented when switching to a new RM system.

In the triad of purely OR-based research, purely behavioral research, and the strategic organizational perspective, we identify a research gap concerning the interplay of analysts and automated systems on the grassroots level. In this area, Bartke et al. (2013) represent an early contribution in pointing out potential difficulties arising from the increasing complexity of RM systems. However, the authors do not consider an empirical example of RM evolution. Based on the field study presented in this paper, we examine the evolving role of analysts when the firm updates the automated RM system and the related processes and structures.

In observing implications from such a switch as it occurs in the real world, we observe a "natural experiment" (Dunning 2012). Other examples of researchers taking advantage of such natural experiments include observing the effects of integrated health information technology on the quality of patient care (Pinsonneault et al. 2017), observing effects in online social networks (Sismeiro and Mahmood 2018) and observing the effects from a change in firm scope (Natividad and Rawley 2016). Given this foundation, findings are primarily limited to the circumstances of the observed firm. However, to our knowledge, this study is the first to consider this perspective in the field of analytics. In doing so, we follow the appeal of BurtonJones and Gallivan (2007) and view the RM process from a multilevel perspective, considering both the individual and the group. In doing so, we hope to provide an example and inspiration for further research in this vein.

\section{Theoretical background}

In the following, we introduce the theoretical background for multilevel analysis following Burton-Jones and Gallivan (2007). Furthermore, we examine task variety and decision authority on the individual level following Brickley et al. (2009, pp. 363-419). We apply the theoretical background introduced in this section to analyze the empirical case in the remainder of this paper. 


\subsection{IS use: differentiating jobs and levels}

Our analysis traces the development of RM analyst jobs based on concepts of decision right assignments and the variety of jobs described in Brickley et al. (2009, pp. 363-419). In this view, a company's value creation process consists of fulfilling a variety of tasks. These tasks are bundled in functions, which contribute to different production steps. In addition, different tasks are bundled into jobs when being assigned to employees. Jobs and functions do not necessarily have to be identical; thus, two different definitions are useful.

We differentiate jobs by variety of tasks and the decision authority. The variety of tasks describes the range of different tasks that are assigned to a job. The decision authority describes where in the hierarchy decisions are made. In addition, we consider jobs as focusing on different groups, e.g., different products or different regions.

\subsubsection{Task variety}

Concerning its task variety, a job can range from being specialized to being broad. A specialized job contains only a single function but is executed for all groups. In contrast to that, a broadly described job contains several functions but is executed for only one group. Both ways of structuring jobs have advantages and disadvantages. Specialized jobs are based on skill, have low training costs, and can efficiently use economies of scale. However, information costs and coordination costs are higher when jobs are specialized, as the transfer of information and interaction between different functions has to be ensured. Furthermore, employees who execute specialized jobs do not have a good overview of the whole process but focus on their own function.

\subsubsection{Decision authority}

Decision rights can be centralized or decentralized. For decentralized decision authority, employees on a lower hierarchical level are allowed to autonomously decide certain issues rather than having to refer to a higher hierarchical level. Advantages of decentralization are local knowledge and rapid decision making, enabling quick responses to market changes. However, this comes with incentive problems and coordination costs due to possible interaction effects of local decision making.

Furthermore, our analysis follows the recommendation of Burton-Jones and Gallivan (2007), who propose a multilevel approach to analyzing the use of IS in organizations. This approach lets us draw a richer and more complex picture of system usage in RM, as it lets us consider implications of both individual use and the joint work of groups of analysts focusing on specific system aspects. Thereby, we can both consider the degree of task variety and responsibilities offered by individual analysts' jobs, and trace fault lines in the communication between groups focusing on different process stages or aspects of RM. 
Burton-Jones and Gallivan (2007) differentiate individual, group, and organizational levels of IS use. The scope of our analysis focuses on individual and group level: on the individual level, we examine individual analysts' jobs and how these jobs correspond to individual process steps implemented by the RM systems. At the group level, we examine how teams of analysts are formed according specific aspects of RM. Based on this differentiation, we discuss the fault lines created by sectioning functions of the RM process across separate groups of analysts and RM systems.

When considering groups of analysts jointly accessing and complementing the automated RM system, we find it especially important to focus on the second guideline stated in Burton-Jones and Gallivan (2007). This guideline points out the relevance of interdependencies in the collective use of a system. Regarding RM systems as SAS, where users necessarily complement the automated system elevates the importance of considering collective versus individual use. Thus, interdependencies between individual users are necessary to refer to system usage as being collective. It is crucial to carve out why interdependencies exist and how they support the overall system function, e.g., improving performance when using the system. We focus on necessary interdependencies in the RM process to analyze the interplay of analysts and automated RM systems.

Collective system usage can be shared or configural Burton-Jones and Gallivan (2007). In shared use, users are homogeneous; everyone uses the system in the same way. In configural use, there is an explicit pattern of users using different functionalities of the system. We apply this lens in the field study to differentiate RM system-analyst interactions.

\subsection{Capacity-based revenue management}

To provide a background to the concept of RM, we first review the system process as described by Talluri and Ryzin (2004). Next, we point out sequential RM functions and related tasks. Lastly, we highlight functional interdependencies and opportunities for group definitions.

As described in Belobaba et al. (2009, pp. 153-181), RM is only one part of the firm's planning process. For the example of airlines, this planning process includes fleet and route planning, departure scheduling and fleet assignment, pricing, RM and sales. The RM system relies on sales data including observed offers and sales as well as on capacity data extracted from the departure schedule. The RM model considered here is a network model, so that demand forecasts and optimized inventory controls apply to network itineraries. In this view, each itinerary can consist of one or more legs (flights), which connect an origin and a destination. The firm may offer different booking classes at different fares on different itineraries.

The model maximizes expected revenue by optimizing the set of offered booking classes to skim the most valuable demand based on a given, fixed capacity. Following the outline of RM functions illustrated by Fig. 1, we define four main RM steps. These are represented by squares in Fig. 1. From left to right, they include pricing, demand estimation and forecast, offer optimization, and inventory. Pricing sets the fare per booking class, while demand estimation and forecasting 


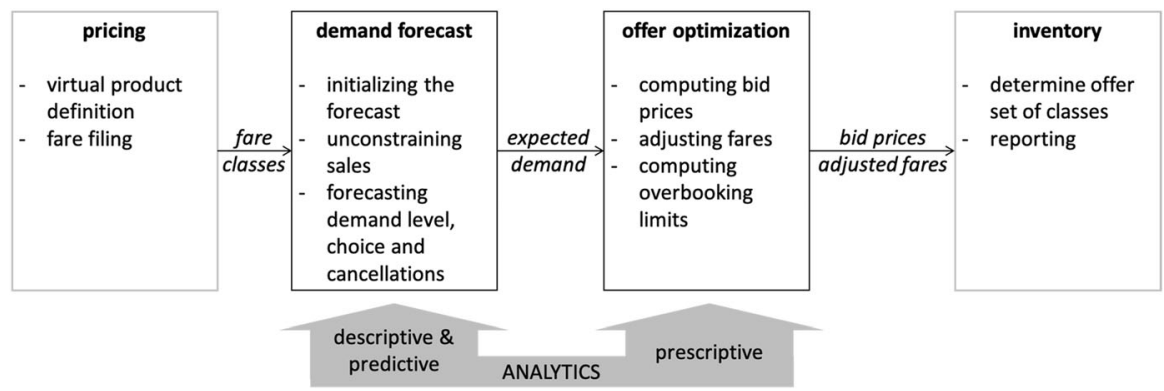

Fig. 1 Revenue management analytics process

parameterize a demand model. Based on the expected demand and the prices, offer optimization prescribes the set of classes to offer at any time. This prescription is implemented in the inventory, which also reports the resulting sales. As indicated in Fig. 1, demand estimation and forecasting solve descriptive and predictive analytics problems, whereas offer optimization exemplifies prescriptive analytics. In the following, we consider these steps as separate functions and further describe specific tasks allocated in these functions. Furthermore, we use this opportunity to point out the role of analysts in each task.

\subsubsection{Pricing function}

Capacity-based RM considers pricing as a preliminary step; this function is mostly executed by manual work with some support from data analysis tools. Pricing includes a "virtual product definition" and a "fare filing" task. Virtual product definition defines booking classes including restrictions (such as weekend stays or refundability). Fare filing assigns one or more fare per booking class. Thus, price setting is not part of the optimization process, but the result of pricing serves as input for forecast and offer optimization. Research and industry visions aim to join pricing and offer optimization in a single dynamic pricing step [compare Fürstenau et al. (2019)]. However, in the case described here, the system update does not implement this.

\subsubsection{Demand estimation and forecast function}

The demand forecast aims to predict the demand per booking class and itinerary as a function of time in the booking horizon and offered classes. To this end, a first task entails estimating the underlying demand from observed sales, which are famously constrained by the offer sets and the limited capacity: customers may have preferred to book a different class, but since it was not offered, they either chose a substitute or did not buy at all. We term this task "unconstraining," following RM conventions. The forecast aims to predict both the number of expected customers in a "demand level forecast" task and their expected choice behavior in a "choice forecast" task. In predicting these parameters, the forecast can consider different 
temporal dimensions, from special events to seasonality to a baseline prediction. Different methodologies take different approaches to operationalizing customer choice-in the example here, it is represented either by buy-down behavior, that means buying a cheaper class as it is available even though a customer would also buy a more expensive class resulting in a loss of revenue, or by demand elasticity. As optimization can include an overbooking function, the forecast also predicts the number of customers who will not utilize the booked service in the "cancellations forecast". Note that some predictive analytics systems accomplish multiple of these tasks in a single step; as a simple example, Holt-Winter's exponential smoothing computes the level, trend, and seasonality of forecasts (Goodwin 2010). While automated algorithms execute all forecasting tasks, human analysts can adjust, e.g., parameters of learning and unconstraining, point out the existence of special events affecting observed demand, and generally adjust the forecast.

\subsubsection{Offer optimization function}

The offer optimization function relies on the forecasted demand as well as information on booking classes, fares, and capacities to determine the best set of classes to offer. Both systems considered in the field study achieve this by computing two parameter sets: bid-prices, and adjusted fares. Therefore, we differentiate the "bid-price" task and the "fare adjustment" task. Through bidprices, the automated optimization algorithm evaluates the opportunity cost of selling one more unit of capacity given resulting limitation in future salable units compared to the expected future demand. The fare of a class has to exceed the bidprice to be available. Human analysts can adjust bid-prices by setting multiplicative or additive factors or upper or lower limits. By adjusting fares, the optimization evaluates the substitution effect resulting when customers can choose between two booking classes and opt for the cheaper alternative. Again, human analysts can adjust the outcome through factors and rules. Furthermore, the optimization function also includes the task of "setting overbooking limits" based on the predicted no-shows and cancellations. Again, automated algorithms compute the initial overbooking limits, but human analysts can adjust them.

\subsubsection{Inventory function}

The inventory implements the optimal offers computed in the offer optimization function by comparing bid-prices and adjusted fares. It only offers booking classes where the adjusted fare exceeds the bid-price. We term this task the "inventory control" task. This task also entails the final opportunity for human analysts to overrule the automated system, as they can limit or extend offers for specific legs of the network. In addition, the inventory records offers and bookings in the "reporting" task. These data are stored in a data warehouse and accessed by the demand estimation and forecast function. Analysts can also complement this step by marking data that should not be used for further iterations, for example, if the data were skewed due to special circumstances such as strikes or international crises. 
Table 1 RM Functions, automated tasks, and manual tasks

\begin{tabular}{lll}
\hline RM function & Automated task & Manual task \\
\hline Pricing & - & Virtual product definition \\
& - & Fare filing \\
$\begin{array}{l}\text { Demand estimation and } \\
\text { forecast }\end{array}$ & - & Initializing the forecast \\
& Unconstraining sales & - \\
& Demand level forecasting & Adjusting the expected demand \\
& Choice forecasting & level \\
& Cancellations forecasting & Adjusting choice parameters \\
Offer optimization & Computing the bid price & Adjusting bid prices \\
& Adjusting fares & Re-adjusting fares \\
& Computing overbooking limits & Adjusting overbooking limits \\
Inventory & Determining offer set of classes & Adjusting offer set of classes \\
& Reporting & Excluding data \\
\hline
\end{tabular}

In theory, there exist automated algorithms for all RM functions. Nevertheless, Table 1 shows that analyst jobs accompany almost every step of the automated system. When documenting and analyzing the field study, we discuss the distribution of tasks into analyst jobs. In terms of multilevel use, attributing tasks to different teams of analysts renders it crucial for them to communicate, just as attributing tasks to different application components in a purely automated system creates the need for joint data access and storage. Therefore, the resulting SAS features two areas where fault lines can occur: within the automated system components and within the multiple levels of human usage. In the field study, we point out changes in the second, as the analysts' organization is adjusted to match the new systems' perspective.

\section{Field study: observing a natural experiment in RM design}

The field study observes a natural experiment in RM design. It focuses on an established airline that offers a complex network of flight itineraries. The airline has been applying revenue management successfully for more than a quarter century. As the firm prepares to overhaul its RM methodology during the field study, it moves between two states, termed cases in the further text: The old versus the new design of the automated system and its embedding in the organization, including analyst jobs.

Specifically, the firm updates its RM systems from implementing a version of the methodology outlined in Fiig et al. (2010) to implementing a version of the methodology outlined in Rauch et al. (2018). Both systems follow the same general logic of capacity-based RM outlined in Fig. 1. However, in their algorithmic logic, the two systems differ according to one crucial aspect: The old system (case 1) 
attempts to predict all aspects of demand at one step based on a single data set. Subsequently, it applies an optimization model that aims to simultaneously exploit both the volume of demand and the expected customers' willingness-to-pay. The new system (case 2) differentiates the problem of predicting the volume of demand and optimizing the allocation of capacity from the problem of predicting customers' willingness-to-pay and optimizing the offer of different fares. The resulting differences in system functions imply different perspectives on RM. Thereby, they motivate different approaches to organizing the human component of the SAS.

The switch excludes the pricing function and tasks related to cancellations and overbookings. Therefore, our analysis neglects these aspects. While different software applications are involved in the RM process, we focus on the methodology in terms of the functions described in the previous section. Defining the RM system as the overall system landscape including all necessary functions lets us analyze the complex effects and interaction in the RM process. We consider the organizational structure by examining the grouping of analyst tasks into the job definitions.

In this section, we first detail the data collection underlying the field study. Subsequently, we list the analyst tasks that we identified in the study. Having established the set of analyst tasks, we describe the before and after cases and detail the grouping of tasks into analyst jobs for each case. Finally, we analyze differences and similarities in the two cases.

\subsection{Data collection}

Table 2 lists the material of the field study as detailed below.

\subsubsection{The site}

This study focuses on a firm that has substantially invested in RM research and has a long tradition of successfully applying state-of-the-art RM. In fact, the firm considers itself an "industry leader" in the field. Both the scale of the considered transport network and the high degree of sophistication in the RM systems create high potential for analyzing the interaction of analysts and systems: smaller and less

Table 2 Overview of collected data

\begin{tabular}{ll}
\hline Interviews & RM analysts (four interviewees) \\
& Head of RM IT (several discussions based on documentation) \\
Internal documentation & System landscape and system definition (old vs. new) \\
& User guide for RM system (old vs. new) \\
& Presentation files (old vs. new) \\
Direct observations & Researcher 1: participated in four analyst workshops \\
& Researcher 1: intern as analyst for three months \\
& Researcher 1: position in IT department over one year \\
& Researcher 2: lead regular training events over four years \\
\hline
\end{tabular}


established firms may suffer from financial restrictions, which either limit the scale of automated RM systems or the size of the skilled workforce. Very small transport providers consider networks that are small enough to make fully manual controls feasible. Furthermore, a long-standing industry cooperation allowed us to observe a switch in the firm's revenue management systems, which creates a unique opportunity to analyze a natural experiment.

\subsubsection{Direct observations}

We gained entrance to the company via a long-standing industry cooperation featuring joint projects executed by doctoral researchers. Based on this, one author (researcher 1) took part in in-house trainings for analyst tasks. By interning as an analyst for three months, she gained hands-on experience in using the relevant RM systems to analyst tasks. Researcher 1 was responsible for monitoring and adjusting the offer optimization and inventory functions for a specific itinerary. In that, she faced similar decisions and challenges as professional analysts in the same position. Following up on this experience, researcher 1 spent more than a year as an observer in the IT department in charge of preparing the system switch. Another author (researcher 2) contributed to periodic company training events based on the old RM system over the course of 4 years. The resulting knowledge and inside contacts shaped the research and findings presented here.

\subsubsection{Interviews}

To validate observations and compare perspectives, we conducted expert interviews with four revenue management analysts. Two of the analysts had responsibilities in pricing and forecasting, while the other two focused on adjusting optimization and inventory parameters. The experience of the interviewed analysts ranged from 2 to 16 years. We chose interview partners for a diversity in tasks and focus as well as with the aim of sampling different degrees of experience.

Our interview approached followed the method of expert interviews described in Meuser and Nagel (2009). We find expert interviews suitable as our aim was to retrieve expert information and knowledge about decision-making, principles, rules and challenges in the job field of an RM analyst. One important prerequisite for expert interviews is a competent interviewer with a certain level of knowledge about the topic, as the focus is not on the interviewee's biography but on strategies and criteria of decision-making. This expertise was given from the researchers' practical and theoretical background in RM.

We conducted semi-structured interviews based on a set of open questions. Interviews were documented through handwritten notes, avoiding, e.g., voice recordings, to emphasize confidentiality. Questions aimed at retrieving knowledge about the analysts' specific tasks, how possible challenges are handled, which systems the analyst uses, and how they communicate with other departments and teams. To provide additional depth, we also asked for illustrative examples. This way we ensured that the interview questions focus on decision-making and action and a "supra-personal level of knowledge" (Meuser and Nagel 2009). 


\subsubsection{Internal documentation}

Finally, the firm provided access to extensive internal documentation on the new and the old system. One part of the material were definitions and visualizations of the system landscape as well as official user guides for some of the systems. This includes information about interfaces and data flows between the pricing, forecasting and optimization units, insights into systems for data processing and consolidation, as well as user interfaces accessed by analysts. Furthermore, we had access to presentation files that describe the old and the new organizational structure of departments and teams, routines, communication guidelines, and expected challenges. The head of the Revenue Management IT department, an experienced expert on IT and RM methodology, supported us in understanding the materials and was available for several discussions based on these materials. Based on these materials, we analyzed the entire RM system landscape, the communication and data transfer between different systems, as well as the type and extent of analystssystem interactions.

\subsubsection{Data analysis}

Our first analysis aims at consolidating the information from the expert interviews and the internal documentation. To do this, we match the analysts' information and knowledge with the corresponding system information. This provides a rich picture of the analysts' job and the interconnection with the RM system landscape. The results are presented and discussed in the following sections.

\subsection{Analyst tasks}

The switch of systems from case 1 to case 2 leaves the general set of tasks unchanged, following the sequence of capacity-based RM as illustrated in Fig. 1. The two cases group tasks in different jobs, while the switch affects the execution of tasks (e.g., by updating the user interface). We list analyst tasks alongside system tasks in Table 1. Our analysis predominantly focuses on tasks that are directly affected by the switch in methodology or, in the case of fare filing, that play a particular role in the design of job definitions. Figures 2 and 3 indicate tasks and

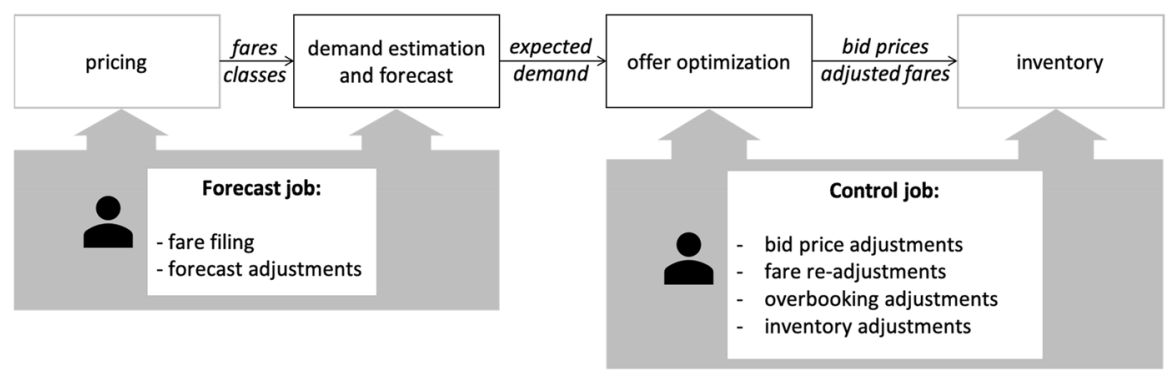

Fig. 2 Case 1-system and organizational structure, functional view 


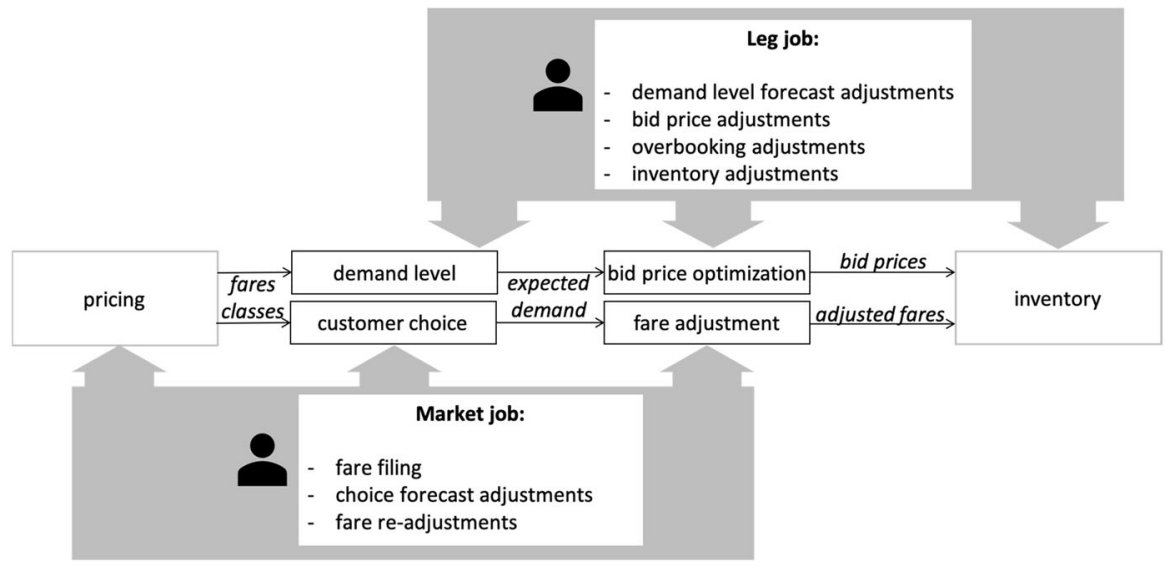

Fig. 3 Case 2-system and organizational structure, process view

their combination to analyst jobs. For example, case 1 combines all tasks related to fare filing and the forecast in a single job.

\subsubsection{Fare filing}

Analysts manually enter fares per booking class and combination of origin and destination into the RM system in a task termed "fare filing." The firm keeps this function predominantly manual, following the idea that pricing decisions rely on expert intuitions about the market, product characteristics, and the firm's strategy. Our interviews reveal that when performing this task, analysts have to consider relevant competing offers and to evaluate the firm's product characteristics, such as departure frequencies and conditions. Prioritizing strategic objectives guides the fare filing task, as analysts, for instance, aim to balance yield and the number of bookings. Pricing also considers information on market and economic developments, politics, and customer expectations. It includes a feedback loop, as analysts set prices, let the RM system control the availability of the related classes, observe ticket sales, and evaluate whether expectations were met. The interviewed experts emphasized that this task affords close cooperation with the sales department for the specific market.

\subsubsection{Initializing the forecast}

When the firm offers a new travel itinerary and has not yet collected sufficient sales data, the automated demand forecast requires some initialization. For this, analysts select a comparable travel itinerary, which they expect to display a similar market behavior. Subsequently, the forecasting system analyzes sales data from this itinerary until sufficient data have accumulated on the new itinerary. 


\subsubsection{Adjusting the forecast: level, choice parameters, and cancellations}

In terms of adjusting the forecast, analyst tasks differ in their temporal focus, pertaining seasonality, events, or the baseline forecast. Analysts can adjust the parameters describing seasonality, indicating, e.g., above-average demand to recur during the summer months. They can also mark single-occurrence and repeat events, such as trade fairs and school holidays, either by just indicating the time frame or by adjusting the demand level or choice parameters for this time frame. When adjusting the baseline prediction, analysts override the expected demand in a lasting fashion due to major changes such as an economic boom or the market entry of a competitor. In doing so, analysts compare the automated forecast's fit with external information and intuition about market behavior. They also analyze forecasts if other groups of analysts report conspicuous availabilities or bid-prices, as these may be caused by a flawed demand forecast. The forecast analysis can include single legs or bundles of itineraries, as well as different booking and departure periods. The interviewed forecast experts state that influencing the forecast brings several challenges: it is not easy to estimate the correctness of the forecast as well as the system's reaction to influences. Furthermore, it is hardly possible to monitor effects because analysts cannot assess with certainty what might have happened without an influence. Because of this, good ex-ante influences are difficult to implement. As the bid-price is computed based on the forecast, analyst adjustments of the forecast affect the offer set optimization, emphasizing the high degree of interrelation between RM process steps.

\subsubsection{Adjusting the bid-price}

The automated system computes the bid-price per leg of the offered transport network to evaluate the current value of left-over capacity in the light of expected demand. The analyst task entails monitoring this parameter and adjusting it in case of systematic errors or sudden changes. To do so, analysts monitor bookings and yield per network leg. They benchmark these indicators on those reported during the previous year to evaluate performance. Bid-price adjustments can be additive or multiplicative factors or upper or lower bounds.

\subsubsection{Re-adjusting fares}

The firm's RM system uses adjusted fares to account for demand dependencies, where customers' choices depend on the set of offered classes. To compensate for buy-down from classes with a high fare when classes with a low fare are offered, the automated offer optimization computes fare adjustments per itinerary and point of sale. Booking classes are only offered if the adjusted fare exceeds the bid-price; if the adjusted fare is negative, a class is never offered. Especially during events, such as holidays and trade fairs, analysts re-adjust these fares manually to override offers. To that end, they can set conditional rules depending on indicators such as days before departure or number of bookings. They can not only change adjusted fares incrementally but also render negative adjusted fares positive. 


\subsubsection{Adjusting overbooking limits}

Based on the forecasted cancellations, the automated offer optimization computes overbooking limits to control the number of tickets to offer in excess of the physical capacity. These overbooking limits represent a compromise between the risk of relinquishing possible revenue from selling a physical seat twice and the risk of having to account for the cost of denying a customer the service. Analysts balance denied boarding costs against the expected revenue by adjusting a set of overbooking parameters. As the physical transport capacity depends on the network leg, overbooking limits are computed per leg.

\subsubsection{Adjusting inventory controls}

A final analyst task includes monitoring the offered classes and the resulting bookings and revenue in the inventory. In this task, analysts can confirm bookings on the waiting list or block particular classes to fine-tune overbooking. They also can limit bookings in specific classes per network leg, independently of itinerary or point of sale. Through this task, analysts implement, for instance, a systematic priorization of loyal customers or adjust to operational failures, e.g., when a flight was cancelled.

\subsubsection{Excluding data}

Finally, to avoid flawed updates to the forecast, analysts can select and exclude sales observations from entering the system. Excluding data can make sense, for instance, when observations were affected by weather conditions or strikes.

\subsection{Case 1: the old system}

\subsubsection{Automated RM system}

In case 1, the automated system consists of two analytics components: the forecast predicts the number of bookings per itinerary and class as well as a share of buydown expected between classes. The optimization computes bid-prices for each network leg and adjusted fares per itinerary and class. The inventory determines the set of offered classes by comparing the current bid-prices and adjusted fares and records bookings.

\subsubsection{Individual level}

As illustrated in Fig. 2 and Table 3, analyst tasks are grouped into two jobs: Analysts that perform the "Forecast" job fulfill both the pricing task and all forecasting tasks. Analysts that perform the "Control" job adjust bid-prices, fares, overbooking parameters, and the inventory. The two jobs are strictly sequential, in that "Forecast" analysts solely work at the beginning of the process and "Control" 
Table 3 Job distribution in case 1 vs. case 2

\begin{tabular}{llllllll}
\hline Case & Job & Pricing & $\begin{array}{l}\text { Demand level } \\
\text { forecast }\end{array}$ & $\begin{array}{l}\text { Customer choice } \\
\text { forecast }\end{array}$ & $\begin{array}{l}\text { Bid- } \\
\text { price }\end{array}$ & $\begin{array}{l}\text { Adjusted } \\
\text { fares }\end{array}$ & Inventory \\
\hline 1 & $\begin{array}{l}\text { Forecast } \\
\text { Control }\end{array}$ & $\mathrm{X}$ & $\mathrm{X}$ & $\mathrm{X}$ & & & $\mathrm{X}$ \\
$\mathrm{2}$ & $\begin{array}{l}\text { Market } \\
\text { Leg }\end{array}$ & $\mathrm{X}$ & $\mathrm{X}$ & $\mathrm{X}$ & $\mathrm{X}$ & $\mathrm{X}$ \\
& & $\mathrm{X}$ & & $\mathrm{X}$ & & $\mathrm{X}$ \\
\hline
\end{tabular}

analysts use their output as input. The firm organization mirrors these jobs in two separate departments.

The "Forecast" job is rather specialized, including few functions, and has a low decision authority, as any influences set in early stages of the process can be easily overruled in later stages. The "Control" job is rather broad, including influences on both leg and itinerary level. Analysts that perform this job have a high decision authority, as they can determine the offer to implement in the inventory.

\subsubsection{Group level}

In this case, it is crucial that analysts that perform the "Control" job communicate with those performing the "Forecast" job: analysts that perform tasks in later steps of the process need to be aware of previous adjustments. Additionally, flawed optimization parameters observed in the later step can be due to a flaw in forecasting, which has to be corrected at the source of the error. Our expert interviews highlight that grouping tasks in a "Control" job is advantageous as decisions that involve both leg and itinerary information can be managed quickly and involve little communication effort. Furthermore, by managing both itineraries and legs of the network, analysts are potentially more aware of the possible decision conflicts.

\subsubsection{Interpretation}

The sequential organization of case 1 emphasizes the collective use of the RM system. Strong interdependencies exist across analysts and their tasks. Furthermore, collective use is configural, as users are specialized and use different parts of the system. Thus, according to Burton-Jones and Gallivan (2007), we can classify the RM system and its organization not only as heterogeneous because user access different system units, but also as stable over time as the usage of systems does not change. A configural approach is plausible when the system, task or users are sufficiently complex. Clearly, the RM system landscape is complex, consisting of several units, interacting with each other and determine the calculation and output of the next one. We also argue that the jobs performed by RM analysts are sufficiently complex even though they are already specialized: analyzing different abstract 
parameters and considering its impacts on outcomes is complicated. Coordination and information exchange with other analysts further complicate the jobs.

The important question is whether this interplay of system and organization supports the overall objective: improving final class availabilities and prices to maximize revenue in situations that cannot be handled alone by the automated system, as well as account for additional strategic goals. The success of this objective seems to predominantly depend on the success of communication between analysts performing the two different jobs. According to our interviews, this communication is time-consuming and often laborious. However, it is clear that at some point functions and responsibilities have to be apportioned as the overall process is too complex and special knowledge is necessary at every step.

\subsection{Case 2: the new system}

\subsubsection{Automated RM system}

In case 2, the newly introduced automated system includes four analytics components, splitting the forecast and offer optimization component each in half. The demand level component of the forecast predicts only the volume of demand, whereas the customer choice component of the forecast predicts demand elasticity. Based on the demand level forecast, the bid-price optimization computes the opportunity cost of capacity. Based on the customer choice forecast, the fare adjustment component adjusts fares to account for potential buy-down between classes. Bid-prices and adjusted fares are combined in the inventory to determine the set of offered classes.

\subsubsection{Individual level}

Case 2 also separates analyst tasks into two different jobs. However, in this case, responsibilities for forecasting and optimization follow a parallel structure (compare Table 3 and Fig. 3): while the "Market" job includes all tasks related to pricing and customer choice on the itinerary level, the "Leg" job includes adjusting the demand level, bid-prices, overbooking parameters, and inventory controls on the leg level.

In its focus on customer choice, the "Market" job has a broad spectrum of tasks, involving the pricing, the forecast, and the optimization component. This job definition aims to bundle the market knowledge of prices and the corresponding price elasticities. Thus, it covers the whole RM process from the angle of itineraries and markets. However, analysts' decisions can still be overruled on the leg level by "Leg" analysts that perform the inventory task. Hence, we consider the "Market" job to have medium decision authority.

The "Leg" job also includes a broad spectrum of tasks, ranging from the forecast over the optimization to the inventory component. As the revenue responsibility is inherent to this job, the decision authority is high. 


\subsubsection{Group level}

In case 2, there also communication and coordination efforts across jobs, even though these are no longer strictly sequential: for instance, consider a case where there appear to be too few classes available on a leg, but the bid-price seems justified. In such a situation, the "Leg" analyst has to confer with the corresponding "Market" analyst to analyze whether any itinerary that uses the leg needs adjustments. Each "Leg" analyst may have to communicate with several "Market" analysts, as a leg can be part of multiple itineraries. At the same time, each "Market" analyst has to communicate with each of the analysts responsible for each of the legs in their itineraries.

\subsubsection{Interpretation}

Our interviews reveal that the analysts look forward to the new organization of responsibility. Leg analysts are now able to influence all demand level controls, from forecast to bid-price to inventory influences. Analysts claim that they can interpret forecasts better with their knowledge of booking curves and bid-prices. Furthermore, less communication is necessary as forecast flaws can be analyzed directly. Still, some leg analysts state that they have the revenue responsibility of the legs but are missing one important availability control without the responsibility for adjusting the customer choice parameter.

In case 2, the collective use of the system is also clearly configural, as one set of users account for the market perspective and the corresponding systems, while the second set of users is responsible for the leg perspective and its systems. Similar to case 1 , the system as well as the tasks of the users are complex, with mathematical algorithms on one side and several parameters to analyze and several decisions to make on the other side. Thus, a configural system usage is reasonable in this case, ensuring the overall goal of maximizing revenue as well as considering other secondary objectives. The RM system and its organization are heterogeneous but stable over time as the usage of systems is stable (Burton-Jones and Gallivan (2007)).

\subsection{Comparative analysis and discussion}

The two cases represent two situations at the same firm, before and after a switch in RM methodology. Notably, the desire to achieve better RM performance motivated the switch in methodology, as management hoped to reduce the variation in adjusted fares and to make the system more accessible and transparent. Table 3 illustrates the resulting shifts in analyst jobs, from a sequential distinction between "Forecast" and "Control" to a more parallel distinction by focus into "Market" and "Leg". It illustrates that while the responsibilities change, the share of responsibilities taken over by each group is still equal. Notably, the overall number of employed analysts also stayed constant.

In case 1, jobs mainly orientate towards functions and align with the RM process: Each job corresponds to a few functions covering a part of the RM process and its 
corresponding units. We term this perspective the functional view. The responsibility for core elements of RM, forecasting an optimization, is distributed across different jobs. This differentiation causes collective and configural system usage, while job definitions and system usage stay constant over time. This way of organizing system usage supports the overall goal of maximizing revenue and other additional objectives. However, the sequential organization creates the risk that analysts focus on their own functions within their individual responsibility while neglecting the overall process success.

Case 2 shows a different approach to both methodology and analysts' responsibility. The new organization emphasizes the separation between the market view and the leg view. "Market" analysts are not only responsible for prices, but also for forecasting customer choice and for monitoring fare adjustments in the optimization. Outcomes from the "Market" and the "Leg" job only combine at the very end of the process, in the inventory, where "Leg" analysts have the final say. Both groups of analysts are responsible for different views of the entire process. Thus, we term this perspective the process view. Even though each job can accompany the whole process, the risk of misunderstandings and lack of insights continues to exist, along a different fault line.

When moving from case 1 to case 2, the firm equalized the degree of task variety across analysts, increasing the range of options for the former "Forecast" analysts. "Market" analyst in case 2 cover a lot more from the RM process, starting from the pricing task, parts of the forecast task, to the fare adjustment task.

In terms of knowledge available to the analysts, case 1 divides forecasting and optimization into different jobs and assigns them to different departments. This divide prohibits "Control" analysts from accessing detailed forecast information, such that they cannot take it into account for decision making. As the demand forecast is the input for revenue optimization, its quality significantly affects revenue performance. Hence, the distribution of responsibilities and access triggers a high need for communication between the analysts performing the different jobs. Operationally, "Control" analysts alert "Forecast" analysts whenever inventory controls do not match their expectations. Our interviews highlighted that this could be challenging, as "Forecast" analysts are not always familiar with the optimization parameters.

The division of information changes in case 2, where analysts that fulfill either job can access both forecast and optimization components. Nevertheless, as "Leg" analysts can still overrule outcomes on the leg level, the main share of responsibility continues to lie in the latest stages of the RM process. In case 2 even more than in case 1, a high degree of communication has to be enforced to avoid conflicts.

Thus, in both cases, there is a potential cut-off in the flow of information, whether it is between "Forecast" and "Control" analyst or between "Leg" and "Market" analysts. In either case, communication and corresponding rules and protocols are necessary to ensure that different departments and different functions work together properly. 


\section{Conclusion: some things change, others stay the same}

This paper uses the opportunity to observe a natural experiment in practical RM in a field study: an airline updates its RM methodology by updating the automated systems and, in consequence, updating the organization of the analysts working to complement the system. We point out the role of automated RM systems as prime examples of analytics, embodying both descriptive, predictive, and prescriptive components. Furthermore, we argue that RM systems are examples of symbiotic analytics systems (SAS), in that they rely on both an automated component and a human component for operational planning. As described in the previous sections, the switch in RM systems affects both the predictive analytics component, i.e. demand forecast, and the prescriptive analytics component, i.e. offer optimization.

Our analysis followed the multilevel approach of Burton-Jones and Gallivan (2007) and considered the job definition on the individual level and system usage and necessary communication effort on the group level. The resulting changes in the analyst organization mirror two different perspectives on the RM process:

In case 1, the firm implements a functional view of the RM landscape: the analyst jobs are organized sequentially, with a high degree of system-oriented specialization. The focus of analyst jobs is on specific RM functions (pricing and forecasting vs. optimization and inventory) and not on the entire process. In case 2 , the firm implements the process view: analyst jobs follow a parallel structure along the entire process. All analysts access both predictive and prescriptive analytics systems, with parallelized influences on customer choice and itinerary-levels and on demand level and leg-levels.

Considering different perspectives on analyst-system interaction helps to understand the underlying process and possible crucial points where communication and coordination are necessary. This role of perspectives may not only apply to RM systems but to all systems that offer heterogeneous interfaces and support configural use. In these cases, firms have to find the best process for an existing organization or acknowledge the need for changing the organization that arises if the IS landscape changes.

We find that RM requires a configural, heterogeneous system usage. In both cases analyzed, analysts can intervene at many different levels: A nudge in the forecast, overwriting optimization parameters for the leg's or itinerary level and finetune overbooking limits at the very end of the process. If in this, RM systems are a good representative for further SAS, design choices have to carefully evaluate implications of offering different levels and types of adjustments. We consider further research on other application fields of SAS, such as workforce scheduling and inventory management, as necessary to verify this idea.

However, the cases differ in the implementation of the system landscape and the organizational structure. The functional view emphasizes the different necessary functions and sets up the system landscape and the organization accordingly. System units and jobs are specialized in one or a few functions risking that the focus does not lay on the overall process. The process view acts the other way around and emphasizes the overall process by splitting the process into two sub-processes that 
can work independently from each other. System units and jobs cover a bigger part of the process making communication necessary between sub-processes. Though in complex systems, tasks always have to be split up making communication and coordination an essential part of the overall success.

A major similarity of both cases is as notable as the differences: Regardless of the state of methodology, the firm considers analysts an inherent part of the system, tasking them with monitoring and giving them the authority to adjust every step of the process. Every outcome of every automated system can be overwritten by analysts so that the analysts' tasks match the system tasks step by step (compare Table 1). While the field study concentrates on a single firm, the design of the relevant software systems, which are marketed to further firms in the industry, reveals that this is idea is widely accepted in practice. Thus, the RM system stayed symbiotic even when the automated component is extended-whether this applies to the majority of SAS or is due to specific characteristics of the investigated firm or the RM domain has to be considered by future research.

The constant importance of analytics shows that the perspective on the RM process determines the organizational as well as the system structure. Only when both structures work hand in hand, the collective use of a complex system like the one in RM can contribute to the success of a company. We cannot say which perspective is better, but this was not the aim of this paper.

Discussions with domain experts after the conclusion of the formal field study interviews revealed the underlying belief that regardless of this organizational decision, updating the automated systems would, eventually, lead to a lower degree in analyst adjustments. While lacking quantitative data to test this expectation, our experience within the RM process makes us doubt this: as assumed flaws and lacks in the automated systems are the main motivation for employing analysts in the first place, analysts have become very skilled at pointing these out. Given the high degree of complexity and uncertainty inherent to RM, a system that fully and automatically adjusts for all possible factors has not yet been described even in the theoretical research literature. Instead, we phrase the following expectation based on the field study's insights: for as long as the RM process and the automated systems allow for analysts to make adjustments, human analysts will continue to have a significant part in RM performance.

If this expectation can be generalized, there arise several caveats for designing SAS. As pointed out in this paper, different job divisions and thereby divisions of responsibility cause different potential fault lines and conflicts in the work of analysts. Communication and configural use turned out to be a crucial element of the "human side" of RM and SAS in general-therefore, systematically supporting these aspects of analyst tasks should be an intrinsic part of systems design.

This study analyzes a change in RM methodology at a single company. The limited number of interviews and their open-ended nature creates some limitations for the resulting insights. However, the ideas produced by this approach could inform further research focusing on a more formal survey aimed at a larger group of analysts, potentially from different firms and industries. The study presented here focused on the definition of jobs and change in system usage. We did not examine possible changes in other important categories such as company culture, perceived 
systems usefulness, or acceptance. All these are important aspects that can also yield interesting insights in the RM domain in particular and SAS in general. Accordingly, comparing the RM system across companies and comparing SAS across domains opens up interesting research opportunities. While our study focused on a large company, differences in company size might also be relevant. Therefore, we encourage for further research in this field regarding other aspects to yield additional insights into this topic.

Open Access This article is licensed under a Creative Commons Attribution 4.0 International License, which permits use, sharing, adaptation, distribution and reproduction in any medium or format, as long as you give appropriate credit to the original author(s) and the source, provide a link to the Creative Commons licence, and indicate if changes were made. The images or other third party material in this article are included in the article's Creative Commons licence, unless indicated otherwise in a credit line to the material. If material is not included in the article's Creative Commons licence and your intended use is not permitted by statutory regulation or exceeds the permitted use, you will need to obtain permission directly from the copyright holder. To view a copy of this licence, visit http:// creativecommons.org/licenses/by/4.0/.Appendix: Questions used to guide the expert interviews

1. Which tasks do you perform in your job?

2. Which systems or system components do you use to execute your job?

3. What are typical examples of situations and circumstances when you have to make a decision?

4. How do you rate your daily work with the systems? Are there recurrent challenges or aspects that complicate your work?

5. With what other teams or departments do you have to communicate and in what kind of situations?

6. What will your future job look like after the implementation of the new system and reorganization?

7. Which challenges and improvements do you expect from your future job definition after the reorganization?

\section{References}

Arnott, D., and G. Pervan. 2005. A critical analysis of decision support systems research. Journal of Information Technology 20: 67-87.

Arvan, M., B. Fahimnia, M. Reisi, and E. Siemsen. 2019. Integrating human judgement into quantitative forecasting methods: A review. Omega 86: 237-252.

Bartke, P., C. Cleophas, and B. Zimmermann. 2013. Complexity in airline revenue management. Journal of Revenue and Pricing Management 12 (1): 36-45.

Bearden, J.N., R.O. Murphy, and A. Rapoport. 2008. Decision biases in revenue management: Some behavioral evidence. Manufacturing and Service Operations Management 10 (4): 625-636.

Belobaba, P., A. Odoni, and C. Barnhart. 2009. The global airline industry, vol. 23. New York: Wiley.

Bendoly, E. 2011. Linking task conditions to physiology and judgment errors in RM systems. Production and Operations Management 20 (6): 860-876.

Bendoly, E. 2013. Real-time feedback and booking behavior in the hospitality industry: Moderating the balance between imperfect judgment and imperfect prescription. Journal of Operations Management 31 (1-2): 62-71. 
Brickley, J.A., C.W. Smith, and J.L. Zimmerman. 2009. Managerial economics and organizational architecture, 5th ed. Burr Ridge: McGraw Hill Education.

Burton-Jones, A., and M.J. Gallivan. 2007. Towards a deeper understanding of system usage in organizations: A multilevel perspective. MIS Quarterly 31 (4): 657-679.

Burton-Jones, A., and C. Grange. 2013. From use to effective use: A representation theory perspective. Information Systems Research 24 (3): 632-658.

Che-Ha, N., F.T. Mavondo, and S. Mohd-Said. 2014. Performance or learning goal orientation: Implications for business performance. Journal of Business Research 67 (1): 2811-2820.

Choi, T.-M., S.W. Wallace, and Y. Wang. 2018. Big data analytics in operations management. Production and Operations Management 27 (10): 1868-1883.

Cleophas, C., D. Kadatz, and S. Vock. 2017. Resilient revenue management: A literature survey of recent theoretical advances. Journal of Revenue and Pricing Management 16 (5): 483-498.

Currie, C.S., and I.T. Rowley. 2010. Consumer behaviour and sales forecast accuracy: What's going on and how should revenue managers respond? Journal of Revenue and Pricing Management 9 (4): 374-376.

Dunning, T. 2012. Natural experiments in the social sciences: A design-based approach. Cambridge: Cambridge University Press.

Dutta, S., M.J. Zbaracki, and M. Bergen. 2003. Pricing process as a capability: A resource based perspective. Strategic Management Journal 24 (7): 615-630.

Evans, J.R., and C.H. Lindner. 2012. Business analytics: The next frontier for decision sciences. Decision Line 43 (2): 4-6.

Fiig, T., K. Isler, C. Hopperstad, and P. Belobaba. 2010. Optimization of mixed fare structures: Theory and applications. Journal of Revenue and Pricing Management 9 (1-2): 152-170.

Fürstenau, D., C. Cleophas, and N. Kliewer. 2019. How do market standards inhibit the enactment of digital capabilities? Business and Information Systems Engineering 30: 1319-1342.

Ghallab, M., D. Nau, and P. Traverso. 2014. The actor's view of automated planning and acting: A position paper. Artificial Intelligence 208 (1): 1-17.

Gönsch, J. 2017. A survey on risk-averse and robust revenue management. European Journal of Operations Research 263 (2): 337-348.

Goodwin, P. 2010. The Holt-Winters approach to exponential smoothing: 50 years old and going strong. Foresight 19: 30-33.

Hart, C., and M. Rafiq. 2006. The dimensions of assortment: A proposed hierarchy of assortment decision making. International Review of Retail, Distribution and Consumer Research 16 (3): 333-351.

Horner, P. 2000. Mother, father of invention produce golden child: Revenue management. OR/MS Today 27: 3.

Kasper, G.M. 1996. A theory of decision support system design for user calibration. Information Systems Research 7 (2): 215-232.

Kocabiyikoglu, A., C.I. Gogus, and M.S. Gonul. 2015. Revenue management vs. newsvendor decisions: Does behavioral response mirror normative equivalence? Production and Operations Management 24 (5): 750-761.

Kocabiyikoglu, A., C.I. Goegues, and M.H. Hekimoglu. 2018. The impact of decision types on revenue management decisions: An experimental study. Decision Sciences 49 (2): 225-249.

Leonardi, P.M., and S.R. Barley. 2008. Materiality and change: Challenges to building better theory about technology and organizing. Information and Organization 18 (3): 159-176.

Liozu, S., and A. Hinterhuber. 2014. Pricing capabilities: The design, development, and validation of a scale. Management Decision 52 (1): 144-158.

Lodree Jr., E.J., C.D. Geiger, and X. Jiang. 2009. Taxonomy for integrating scheduling theory and human factors: Review and research opportunities. International Journal of Industrial Ergonomics 39 (1): 39-51.

Meuser, M., and U. Nagel. 2009. The expert interview and changes in knowledge production, interviewing experts. research methods series, 17-42. New York: Palgrave Macmillan.

Mukhopadhyay, S., S. Samaddar, and G. Colville. 2007. Improving revenue management decision making for airlines by evaluating analyst-adjusted passenger demand forecast. Decision Sciences 38 (2): $309-327$.

Natividad, G., and E. Rawley. 2016. Interdependence and performance: A natural experiment in firm scope. Strategy Science 1 (1): 12-31.

Orlikowski, W.J. 2001. Technology and institutions: what can research on information technology and research on organizations learn from each other? MIS Quarterly 25 (2): 145-165. 
Pinsonneault, A., S. Addas, C. Qian, V. Dakshinamoorthy, and R. Tamblyn. 2017. Integrated health information technology and the quality of patient care: A natural experiment. Journal of Management Information Systems 34 (2): 457-486.

Rauch, J., K. Isler, and S. Poelt. 2018. Disentangling capacity control from price optimization. Journal of Revenue and Pricing Management 17 (2): 48-62.

Schütze, C. and Cleophas, C. 2019. Revenue management decision-making given non-stationary demand and two alternative decision tasks. Working paper on SSRN.

Sismeiro, C., and A. Mahmood. 2018. Competitive vs. complementary effects in online social networks and news consumption: A natural experiment. Management Science 64 (11): 5014-5037.

Smith, B.C., J.F. Leimkuhler, and R.M. Darrow. 1992. Yield management at American airlines. INFORMS Journal on Applied Analytics 22 (1): 8-31.

Sprague, R. 1980. A framework for the development of decision support systems. MIS Quarterly 4 (4): $1-26$.

Strong, D., and O. Volkoff. 2010. Understanding organization-enterprise system fit: A path to theorizing the information technology artifact. MIS Quarterly 34 (4): 731-756.

Talluri, K., and G. Ryzin. 2004. The theory and practice of revenue management. Berlin: Springer.

Wang, S. \& Yeoh, W. 2009. How does organizational culture affect IS effectiveness: A cultureinformation system fit framework. In 2009 international conference on electronic commerce and business intelligence, 67-70.

Wanyama, I., and Q. Zheng. 2011. The fit between organizational structures and IS implementation: The case of IFMIS in Kenya. International Journal of Trade, Economics and Finance 2 (2): 131-137.

Wild, T. 2017. Best practice in inventory management. London: Routledge.

Zeni, R. 2003. The value of analyst interaction with RM systems. Journal of Revenue and Pricing Management 2: 37-46.

Publisher's Note Springer Nature remains neutral with regard to jurisdictional claims in published maps and institutional affiliations. 